\title{
Commentary: Is off-pump technique a safer procedure for coronary re- vascularization? A propensity score analysis of 20 years of experience
}

\author{
Federico Paredes Vignoli* \\ Cardiac Surgery Department, Hospiten Rambla, Santa Cruz de Tenerife, Spain
}

Article Info

\section{Article Notes}

Received: November 16, 2017

Accepted: December 28, 2017

\section{*Correspondence:}

Dr. Federico Paredes Vignoli, Cardiac Surgery Department, Hospiten Rambla, Santa Cruz de Tenerife, Spain; Email: feco_py@hotmail.com

(c) 2017 Paredes F. This article is distributed under the terms of the Creative Commons Attribution 4.0 International License.
Coronary artery bypass grafting with extra corporeal circulation (ONPCABG) has been considered the "gold standard" for the surgical treatment of is chemic heart disease. It is known the inflammatory process triggered in the organism by the extra corporeal circulation, as well as them morbidity associated to it.

Coronary artery bypass grafting without extra corporeal circulation (OPCABG) is initiated in order to reduce aggression to the organism, avoiding the use of the extra corporeal circulation machine. Despite the growing development and acceptance that the technique has had, its advantages as well as indications are not well established.

Randomized prospective studies have been designed comparing it with the traditional technique with extracorporeal circulation, most of them don't found advantages, unlike, some disadvantages were described, such as a significant decrease in the number of grafts performed, as well as a lower permeability of them ${ }^{1}$.

Several meta-analyzes have shown a significant reduction in in-hospital morbidity and mortality in patients operated without extracorporeal circulation. However, the disadvantages persist in terms of the number of grafts and their permeability, associated with a higher rate of repeated revascularization and lower longterm survival ${ }^{2-6}$.

Our work presents the experience of 20 years of development of OPCABG, comparing the results with patients operated ONPCABG during the same period of time. Data were collected from 3097 patients, adequate volume to draw conclusions, $43 \%$ were operated without extra corporeal circulation, what show the extensive experience with this technique. As it is a retrospective work, a propensity score matching was carry out in order to equalize the characteristics of the samples and make a more adequate comparison. 2 groups of 1004 patients with similar pre-surgical characteristics were obtained.

30 -day mortality was similar in both groups, $2.8 \%$ vs $3.8 \%$ for the OPCAB and ONPBAC group respectively. This not coincide with the latest meta-analyzes published, which highlight a lower in-hospital mortality in patients operated without extracorporeal circulation ${ }^{4-6}$. However, in our experience, we demonstrate noninferiority in terms of immediate mortality with respect to traditional 
technique. Interesting data have been the significant decrease in postoperative complications. Respiratory complications decreased in their entirety, which could be justified by avoiding the pulmonary collapse associated with extracorporeal circulation. Among the hemodynamic complications, the appearance of postoperative atrial fibrillation marked the difference between both techniques. This coincides with the results of current studies that shown the inflammatory response as an important factor and predictor of the appearance of postoperative atrial fibrillation, which in theory would be reduced by avoiding extracorporeal circulation. Regarding neurological complications, surgery without extracorporeal circulation is aimed at reducing the manipulation of the aorta, at least avoiding the total clamp of it, depending on the specific technique, as partial clamp may be necessary to perform proximal anastomoses, and in this way avoid the risk of embolism by calcium plaques. In this work, a significant reduction in neurological complications with OPCABG was found, from mild ones as disorientation and agitation episodes, to the most devastating ones, such as established ictus.

All these complications are associated with a prolonged hospital stay, which explains the difference in the Intensive Care Unit and total hospital stay in favor of patients operated without extracorporeal circulation, which it also has a very important economic impact.

One of the weak points that have been found with this technique is a significant decrease in the long-term survival. The main causes could be the difference in the number of grafts performed with each technique, as well as the lower permeability in the medium and long term and the consequent higher incidence of repeated revascularization associated with surgery without extracorporeal circulation. In this work a lower mean of grafts performed was found in the OPCABG group, this could be explained because this group includes patients from the beginning of application of the technique with its consequent learning curve and less available technical material. Likewise, practically all patients under go isolated revascularization of the anterior descending artery are includes in the OPCABG group which plays an important role when the mean number of grafts performed were calculating. However, one of the most important contributions of this work is the long-term follow-up, with a mean of $7.4 \pm 4.3$ years for the OPCABG group and $9.4 \pm 4.2$ years for the ONPCABG group, finding no significant difference in all cause mortality $(12.3 \%$ vs. $12.9 \% \mathrm{p} 0.42$ ) and survival during the period studied. Unlike the results presented in the meta-analyzes, in which the long-term survival is greater in patients operated with extracorporeal circulation, this work includes patients from a single center, reducing the variability in terms of surgical criteria and techniques used, as well as in the pre and postoperative management of patients, which makes them more comparable. A relevant fact, to which there was no access, was the repeated revascularization rate on our group of patients.

One of the news with respect to the other work is the uni and multivariant analysis of the factors that predict mobility and mortality. These data suggest that extracorporeal circulation would compromise immediate postoperative results, as it is a positive predictor. In addition, is known that chronic obstructive pulmonary disease and renal failure, also positive predictors of morbidity and mortality, are closely associate with complications after extracorporeal circulation.

Currently, OPCABG has been widely disseminated, with its advocacy and detractors groups. However, with the evidence presented until now, it is difficult to find its advantages and exact indications. Some studies aim to demonstrate its efficacy and safety in octogenarian patients ${ }^{7,8}$, based on the excellent immediate postoperative results by reducing in-hospital morbidity and mortality. One of the most recent prospective trials (CORONAY) have report the outcomes after 5 years of follow-up. There were no significant differences between the off-pump group and the on- pump group in the rate of the composite outcome of death, stroke, myocardial infarction, renal failure or repeat revascularization; also, there were no differences in quality of life measures between groups ${ }^{9}$. This is interesting and could have an important clinical implication at the time of selecting the appropriate patients to undergo OPCABG based on this results, taking into account the excellent results after 5 years of follow-up and the life expectancy of the octogenarian patients.

On the other hand, with the development of minimally invasive cardiac surgery, OPCABG becomes a weapon that can be associated with this procedure, increasing its advantages and benefits for patients.

For all the above, it is important that serious work groups present, their current results with this technique, especially once the learning curve has been overcome and taking advantage of all the devices currently used to provide security to the technique.

\section{References}

1. Shrover L, Grover D, Hattler B, et all. On-Pump versus OffPumpCoronary-Artery Bypass Surgery (ROOBY). N Engl J Med. 2009; 361: 1827-37.

2. Hayashi Y, Maekawa A, Sawaki S, et all. Long-termpatency of on- and off-pumpcoronaryartery bypass graftingwith bilateral internalthoracicarteries: thesignificance of latestringsigndevelopment in the off-pumptechnique. InteractCardioVascThoracSurg. 2017; 25: 799-805.

3. Takagi H, Mizuno Y, Niwa M, et al. A meta-analysis of randomizedtrialsforrepeatrevascularizationfollowing off-pump versus on-pumpcoronaryartery bypass graftingInteractiveCardioVascular and ThoracicSurgery. 2013; 17: 878-881. 
4. Filardo G, Hamman BL, da Graca B, et all. Efficacy and effectiveness of on- versus off-pumpcoronaryartery bypass grafting: A meta-analysis of mortality and survival.J ThoracCardiovascSurg. 2017; (17)31: 7865 .

5. Takagi $\mathrm{H}$, Ando $\mathrm{T}$ andMitta $\mathrm{S}$. Meta-AnalysisComparing $\geq 10$-Year Mortality of Off-Pump Versus On-PumpCoronaryArtery Bypass Grafting. Am J Cardiol. 2017; (17)31: 377-2.

6. Takagi H, Matsui M, Umemoto T. Off-pumpcoronaryartery bypass mayincrease late mortality: a meta-analysis of randomizedtrials. AnnThoracSurg. 2010; 89(6): 1881-8.
7. Khan $H$, Uzzaman $M$, Benedetto $U$, et al. On- or offpumpcoronaryartery bypass graftingforoctogenarians: A metaanalysis of comparativestudiesinvolving 27,623 patients.Int J Surg. 2017; 47: 42-51.

8. Yuksel A, Yolgosteren A, Kan II, et all. A comparison of earlyclinicaloutcomes of off-pump and on-pumpcoronaryartery bypass graftingsurgery in elderlypatients.Acta ChirBelg. 2017; 25: 1-6.

9. Lamy A, Deveraux P.J, Prabhakaran D, et al. Five-Year Outcomes after Off-Pump or On-Pump Coronary Artery Bypass Grafting. N Engl J Med. 2016; 375: 2359-2368. 